\title{
Transforming Upper-Division Quantum Mechanics: Learning Goals and Assessment
}

\author{
Steve Goldhaber, Steven Pollock, Mike Dubson, Paul Beale and Katherine Perkins
}

Department of Physics, University of Colorado, Boulder, Colorado 80309, USA

\begin{abstract}
In order to help students overcome documented difficulties learning quantum mechanics (QM) concepts, we have transformed our upper-division QM I course using principles of learning theory and active engagement. Key components of this process include establishing learning goals and developing a valid, reliable conceptual assessment tool to measure the extent to which students achieve these learning goals. The course learning goals were developed with broad faculty input, and serve as the basis for the design of the course assessment tool. The development of the assessment tool has included significant faculty input and feedback, twenty-one student interviews, a review of PER literature, and administration of the survey to two semesters of QM I students as well as to a cohort of graduate students. Here, we discuss this ongoing development process and present initial findings from our QM class for the past two semesters.
\end{abstract}

Keywords: physics education research, quantum mechanics, assessment

PACS: 01.30.la,01.40.Fk,01.30.Cc,01.40.Di

\section{INTRODUCTION}

For the last decade, there has been a new focus in physics education research aimed at undergraduate quantum mechanics (QM).[1] In recent years a growing body of evidence has shown that students experience a number of difficulties learning upper-division QM[2, 3]. In order to address these difficulties, the physics department at CUBoulder, using findings from lower-division PER[4] as well as early results from other reform efforts[3], initiated an effort to reform the QM I course. Normally taken by 35 - 50 junior physics and engineering physics majors, this course is the first part of a two-semester sequence. Topics include the Schrödinger equation, 1-D solutions, operator methods, angular momentum and the hydrogen atom. Classroom instruction using the new methods began in the Spring 2008 semester. Since then, the following changes have been made to the class structure:

- The authors and colleagues have developed over 250 concept test questions and other activities to promote student engagement during lectures.[8]

- The course has adopted an optional weekly recitation session where students work through a tutorial in small groups. Each hour-long session is facilitated by the course instructor and a learning assistant.[5] A few of the tutorials were developed by the authors with the majority coming from the University of Washington.[3, 6]

- Homework problems were augmented to include more justification of reasoning, estimation, and tasks requiring students to make connections between mathematical representations, physical meaning and other representations (e.g., graphical).[7]
- Instructor office-hours were replaced with a cooperative, weekly session where students worked in groups on the homework.

In addition to the changes outlined above, two other initiatives helped to focus the reform efforts as well as to track student achievements and learning difficulties: course learning goals and an assessment tool.

\section{LEARNING GOALS}

In the Summer of 2008, we began the process of creating learning goals for our QM I course by interviewing faculty who had experience teaching this course as well as a few faculty who had taught the preceding and following courses in the sequence. These interviews were followed by a series of meetings with interested faculty members who discussed learning goals both for specific course content and for general skills important to faculty. Overall, eighteen faculty members contributed their time and expertise to this process. An example of a contentspecific goal is "Given a wave function and an observable operator, students will be able to calculate that operator's expectation value" while an example of a general skilloriented goal is "Students should be able to sketch the physical parameters of a problem (e.g., wave function, potential, probability distribution), as appropriate for a particular problem.’[8]

While the learning goals developed during the process described above cover much of what is normally taught in an upper-division quantum mechanics course, it became apparent, both in the interviews and in the group meetings that there are certain areas of both the pedagogy and the interpretation of quantum mechanics where physics faculty have widely divergent views. This situation complicates efforts to reform instruction in quantum 
mechanics and is discussed in another paper.[9]

\section{QUANTUM MECHANICS ASSESSMENT TOOL (QMAT)}

Formal classroom assessments (i.e., exams) often do not explicitly probe students' conceptual understanding or sense-making skills in a consistent manner.[10] And yet, these are among the key learning goals identified by faculty for upper-division QM. Therefore, as a major component of this effort, we have created a conceptuallyfocused assessment tool which is described below.

\section{Goals of the QMAT}

The assessment tool was developed with three goals in mind: reflect faculty learning goals, provide an assessment of student learning difficulties, and act as a tool to help guide faculty efforts at improving QM instruction.

Reflect Course Learning Goals: For the QMAT to be useful to and valued by faculty, its questions need to measure student learning of content and skills which most faculty view as important and feel their students should master.

Assess student learning difficulties: Several researchers have found persistent learning difficulties experienced by students undertaking the formal study of quantum mechanics.[2, 3, 11-15] Therefore, the QMAT should measure the extent to which students are still experiencing these difficulties after the course.

Assist faculty in course improvement: Physics faculty are currently engaged in efforts to reform QM instruction at many colleges and universities. By comparing data across semesters and at different institutions, faculty should be able to use the QMAT as a tool to aid their course-improvement efforts.

\section{Development Process}

The questions on the QMAT were developed based on faculty input, adapted from published assessment questions, or inspired by observed student difficulties.[3, 16, 17]. Students who had previously completed the course were interviewed as they worked through these questions. Revisions were made and a final set of sixteen questions were selected after a total of thirteen student interviews. This preliminary version was administered during the last week of class in the Fall 2008 semester. Students were given one fifty-minute class period to complete the QMAT. While the assessment was optional, students were encouraged to try their best and were given feedback on their performance in different subject areas (e.g., probability density, time development). Twentyseven (out of a total of thirty-three) students took the QMAT.
After the results were analyzed, we revised several of the questions and replaced others. Thirteen more student interviews were conducted in order to assess question validity (eight interviews) and to learn more about student thinking (five interviews). Finally, faculty were consulted both in a working group and via an email survey after which a final set of 14 questions was selected.

This new QMAT version[8] was administered in a similar fashion near the end of the Spring 2009 semester to 36 students (46 were registered for the course). The class was taught by a non-PER faculty member using the materials and reforms mentioned above.

\section{Results and Discussion}

The QMAT contains questions addressing several areas of the quantum mechanics curriculum. Figure 1 shows student performance in five categories of learning goals which are probed by multiple questions. During our analysis of the exams, student learning difficulties were particularly apparent in the areas of measurement and time evolution. These difficulties, along with sample questions, are discussed below.

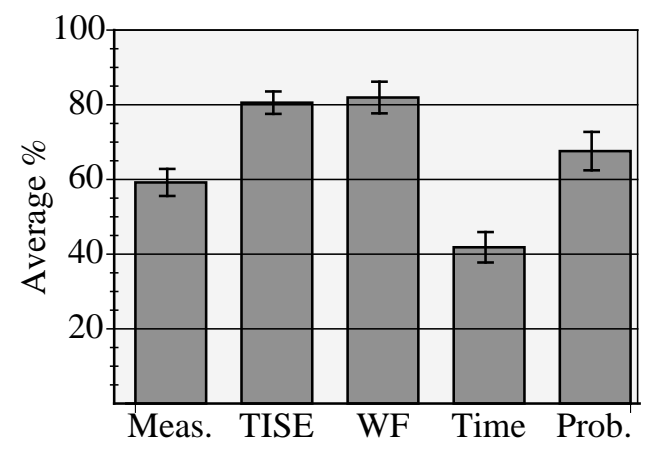

FIGURE 1. QMAT scores by learning-goal category for the Spring 2009 semester. The categories are: measurement (Meas.), the time independent Schrödinger equation (TISE), wave functions / boundary conditions (WF), time evolution (Time), and probability / probability density (Prob.). Error bars indicate the standard error of the mean.

\section{Student difficulties with measurement}

Several researchers have noted student difficulties working with measurement in quantum mechanics.[3, 14, 1820] The QMAT is designed to probe student learning in this area with parts of three different questions.

Parts (a) and (b) of the question shown in Figure 2 measured significant confusion about the state of a particle in an infinite square well after a series of incompatible measurements. $72 \%$ of students correctly noted that the wave function after the first measurement is $u_{2}(x)$, however, only $31 \%$ recognized that all allowed energies were possible after a subsequent position measurement (35\% of the students who correctly answered part (a)). A 
Consider a particle in a $1 \mathrm{D}$, infinite square well with width $a$, centered at $a / 2$. The normalized energy eigenstate wave functions are $u_{n}(x)$ with energies $E_{n}$ ( $n=1$ is the ground state).

The particle starts in a state given by

$$
\Psi(x, t=0)=\left(\sqrt{\frac{4}{5}} u_{1}(x)+\sqrt{\frac{1}{5}} u_{2}(x)\right) .
$$

a) You make an energy measurement on this system and find the maximum possible value for the energy. What is the state, $\psi(x)$, of the system after this measurement?

(72\% correct)

b) After the energy measurement, you make a position measurement. After this position measurement, you immediately re-measure the energy. At this point, what value(s) could you get for energy?

(31\% correct)

\section{c) Does your answer to part b depend on how long you wait between the position and en- ergy measurements? Explain. \\ (11\% correct)}

FIGURE 2. Measurement problem from QMAT. This question builds on work from ref. [3] (p. 409) and from ref. [15]

common error in part (a) was the choice of the lower energy state because it had a higher probability. The common errors on part (b) - from students who said that the choices were $E_{1}$ or $E_{2}$ (42\% of all answers) and from students who said or implied that the position measurement would not alter the energy (19\%) - reveal that students are not fully grasping the important relationships between energy, a particle's wave function, and measurement.

Students responses to statement I in Figure 3 demonstrate that many students fail to properly grasp the distinction between the Hamiltonian operator and physical measurement - only $28 \%$ were able to explain the difference satisfactorily. Two common answers among the $61 \%$ of students who incorrectly agreed that acting on a quantum state with the Hamiltonian is mathematically equivalent to measuring the energy were: 1) because $\hat{H}|\psi\rangle=E|\psi\rangle(32 \%)$, and 2) a statement to the effect that any quantum state has a well-defined energy which is what is measured when the Hamiltonian is acted on that state $(23 \%)$.

\section{Student difficulties with time development}

Another area where researchers have documented student difficulties is in the area of time development.[3,
Which of the statements below are true for any quantum state, $|\psi\rangle$ (Circle either $\mathbf{T}$ (the statement is always true) or $\mathbf{F}$ for each statement)?

I. $\mathbf{T}$ or $\mathbf{F}$ : Acting on $|\psi\rangle$ with the Hamiltonian is the mathematical equivalent of making a measurement of the energy of that state. (28\% correct)

II. T or F: Applying the Hamiltonian to $|\psi\rangle$ gives you information about how that state will evolve in time.

(25\% correct)

FIGURE 3. True / False questions from QMAT. Statement I was adapted from ref. [17]

14, 20] The QMAT looked at student learning in this area with 2 problems and in parts of 2 others.

Part (c) of the problem in Figure 2 exposed student difficulties in working with the time development of quantum superposition states by asking students if waiting before making an energy measurement can affect the possible measured energies. Only 4 students $(11 \%)$ managed to convincingly describe why the amount of time before the second energy measurement did not matter (only 3 of whom earned full credit on the rest of the problem). Among students who felt that the time delay would matter, the most common reasons were that the energy eigenstates would evolve at different speeds or that the delta function resulting from the position measurement would spread out which would change the energy of the state. A different group of 4 students who felt that the position measurement did not change the fact that the system was in an energy eigenstate (part (b)) thought that time did not matter because it was a stationary state. In the words of one student: "No. Measuring $E$ sets the wave eq with that val forever. Collapses it to that $u_{n}$." Students who felt that time does not matter are not making use of the unique role played by energy in QM as shown by this representative answer; "No. regardless of how long you wait the wavefunction will have collapsed to an eigenvector of position space."

Statement II in Figure 3 demonstrated that students have difficulties with the Hamiltonian operator's role in the time development of quantum states. Only $44 \%$ correctly agreed that the Hamiltonian operator is associated with time development and only $25 \%$ wrote a convincing explanation (mostly appealing to the full, timedependent Schrödinger equation or breaking the wave function into energy eigenstates and multiplying each term by the appropriate time-dependence term). The students who disagreed with the statement seem to draw no connection between the Hamiltonian and the full Schrödinger equation saying things like: " $H|\psi\rangle$ tells you 
nothing about time", "The Hamiltonian gives information about energy only", "when we use $\hat{H}$, it's to solve the TISE, so $\hat{H}$ doesn't really tell us about time.", and " $\hat{H}=\frac{\hat{p}^{2}}{2 m}+V$ this has no time dependence."

Student concepts on time development of quantum states are further probed by the question in Figure 4.

Is the following statement true for all operators, $\hat{Q}$ ? Explain briefly why you agree or disagree. A system which is in an eigenstate of $\hat{Q}$ will stay in that state until disturbed by measurement. $(36 \%$ correct)

FIGURE 4. QMAT question about time development of an eigenstate

Only $36 \%$ of students gave a convincing explanation of why the statement was not true for an arbitrary operator (usually either a counter example or a statement that it is only true for energy eigenstates[21]). Among students who agreed with the statement (43\%), the two most popular reasons were that it was a postulate of QM or that it was true simply because it was an eigenstate. A representative quote from the latter category is: "Yes, Eigenstate $=$ stationary state". Meanwhile $15 \%$ of the students who disagreed with the statement indicated that nothing could be said until a measurement of $\hat{Q}$ was made. For instance, one student wrote "Until measurement is made, we don't know what state the system is in. (accompanied by a picture of a cat in a box)".

\section{CONCLUSIONS}

These results demonstrate that the QMAT functions as an assessment tool by exposing common student difficulties in areas such as measurement and time development in QM. It also demonstrates that students in CU's QM I course are not achieving all of our learning goals, despite reforms that include clicker questions and other interactive techniques targeting these ideas. Thus, the QMAT can serve to raise faculty awareness of student difficulties and guide future reform efforts.

Specifically, we find that across the QMAT, students frequently respond as though: all quantum states (including superposition states) have a definite energy, and time dependence only requires 'tacking on' a single term $\exp [-i E t / \hbar]$ to any quantum state (including superposition states). These observations are consistent with existing QM research literature.[2, 3, 14, 15] Our preliminary interviews suggest that students are overgeneralizing from the time independent Schrödinger equation, $\hat{H}|\psi\rangle=E|\psi\rangle$. Students also frequently respond as though sequential measurements on a quantum state retain all original information encoded in the starting state, again consistent with literature.[3]
Moving forward, the QMAT will continue to be used at CU and is available to faculty interested in administering the test to their QM classes. These results, when pooled, will aid the community in the search to find effective new approaches to teaching upper-division QM.

\section{ACKNOWLEDGMENTS}

We thank Carl Wieman and Sam McKagan for useful discussions. We also thank the physics faculty members who generously contributed to the creation of both the learning goals and the QMAT: A. Becker, T. DeGrand, O. DeWolfe, N. Finkelstein, C. Greene, A. Hasenfratz, E. Kinney, K. Mahanthappa, U. Nauenberg, J. Price, C. Rogers, K. Stenson, and E. Zimmerman. This work is funded by The CU Science Education Initiative and NSF-CCLI Grant \# 0737118.

\section{REFERENCES}

1. B. A. Thacker, Editor, H. S. Leff, Editor, D. P. Jackson, and Editor, Am. J. Phys. 70, 199-367 (2002).

2. C. Singh, 2006 Phys Ed PERC Proc, AIP, 883, pp. 185-188 (2007).

3. A. D. Crouse, Ph.D. thesis, University of Washington (2007).

4. L. C. McDermott, and E. F. Redish, Am. J. Phys. 67, 755-767 (1999).

5. V. Otero, N. Finkelstein, R. McCray, and S. Pollock, Science 313, 445-446 (2006).

6. P. Shaffer, Private communication (2008).

7. N. S. Podolefsky, and N. D. Finkelstein 2007 PERC Proc, AIP, 951, pp. 164-167 (2007).

8. The full set of learning goals, assessments, and other course materials, are available at www.colorado.edu/sei/departments/physics_3220.htm.

9. M. Dubson, S. Goldhaber, S. Pollock, and K. Perkins, "Faculty Disagreement about the Teaching of Quantum Mechanics," in 2009 PERC Proc, AIP, 2009.

10. G. Gibbs, and C. Simpson, Learning and Teaching in Higher Education 1, 3-31 (2004).

11. B. S. Ambrose, Ph.D. thesis, University of Washington (1999).

12. D. Domert, C. Linder, and Å. Ingerman, Eur. J. Phys. 26, 47-59 (2005).

13. H. Sadaghiani, and L. Bao, 2005 PERC Proc, AIP, 818, pp. 61-64 (2006).

14. C. Singh, Am. J. Phys. 69, 885-896 (2001).

15. C. Singh, Am. J. Phys. 76, 277-287 (2008).

16. E. Cataloglu, and R. W. Robinett, Am. J. Phys. 70, 238-251 (2002).

17. E. Gire, and C. Manogue, 2008 PERC Proc, AIP, 1064, NY, pp. 115-118 (2008).

18. D. F. Styer, Am. J. Phys. 64, 31-34 (1996).

19. H. R. Sadaghiani, Ph.D. thesis, The Ohio State University (2005).

20. C. Singh, 2004 PERC Proc, AIP, 790, pp. 23-26 (2005).

21. While this is only true for some QM systems, students were given credit for this type of answer. 